\title{
A quantitative study of IMRT delivery effects in commercial planning systems for the case of oesophagus and prostate tumours
}

\author{
J. Seco ${ }^{(1}$, C.H. Clark ${ }^{(1}$, P.M. Evans ${ }^{(2}$ and S. Webb ${ }^{(2}$ \\ ${ }^{1}$ Physics Department, Royal Marsden NHS Trust \\ Fulham Road London SW3 6JJ \\ ${ }^{(2}$ Joint Department of Physics, Institute of Cancer Research \\ and Royal Marsden NHS Trust \\ Downs Road, Sutton Surrey SM2 5PT
}




\begin{abstract}
This study focuses on understanding the impact of IMRT delivery effects on commercial treatment-planning systems such as Pinnacle (ADAC Laboratories Inc.) and CadPlan/Helios, (Varian Medical Systems) planning systems. The study was performed for tumours of the oesophagus and prostate and pelvic nodes. The oesophagus was planned with the ADAC planning system assuming IMRT delivery, via multiple static fields (MSF) or compensators, using the Elekta SL25 with a MLC component. While, for the prostate and pelvic nodes IMRT planning was performed with Cadplan/Helios assuming a dynamic delivery (DMLC) using the Varian 120-leaf Millennium multileaf collimator (MLC).

In a commercial planning system, since IMRT delivery effects are not included into the optimisation process, fluence renormalisation is required such that the mean delivered PTV dose equals the initial prescribed PTV dose. The study was divided into two main parts: (a) analysing the dose distribution within the PTV, produced by each of the delivery techniques, after the delivered fluence has been renormalised such that all IMRT techniques have their mean PTV dose equal to the initially prescribed PTV dose; and (b) studying the impact of the IMRT delivery technique on the surrounding critical organs such as the spinal cord, lungs, rectum, bladder etc.

In preparing the optimum fluence profile for delivery, the PTV dose has been "smeared" by the IMRT delivery techniques. In the case of the oesophagus, the critical organ spinal cord received a significantly greater dose than initially planned, due to the delivery effects. The increase in the spinal cord dose is of the order of 2-3 Gy. In the case of the prostate and pelvic nodes, the IMRT delivery effects led to an increase in approximately 2 Gy in the dose delivered to the secondary PTV, the pelvic nodes. In addition to this, the small bowel, rectum and bladder have received an increased dose of the order of 2-3 Gy to $50 \%$ of their total volume.
\end{abstract}


IMRT delivery techniques strongly influence the delivered dose distributions for the oesophagus and prostate/pelvic nodes tumour sites and these effects are not accounted for in the Pinnacle (ADAC Laboratories Inc.) and the CadPlan/Helios, (Varian Medical Systems) planning systems. 


\section{Introduction}

The treatment planning of carcinoma of the oesophagus is particularly difficult, due to the location of the tumour and surrounding critical organs. The planning target volume (PTV) is situated in close proximity to the spinal cord and is, usually, completely surrounded by the lungs, a radiosensitive organ.

Surgery remains the main treatment for carcinoma of the oesophagus, despite the long-term survival rates following oesophagectomy being poor. Radiotherapy is commonly used for primary tumours of the oesophagus that are deemed unsuitable for surgery. In particular, in the upper third of the oesophagus, radiotherapy may be considered as the treatment of choice. The 3 and 5 year survival figures following radiotherapy obtained at Christie Hospital by Sykes et al (1998) were 27 \% and 21 $\%$, respectively. In addition to radiotherapy alone, the combination of high-dose radiation therapy with chemotherapy may improve the survival rate and local tumour control (Al-Sarraf et al 1994 and Smith et al 1998). As shown in these reports, the addition of chemotherapy has been shown to improve survival significantly, with corresponding improvements in loco-regional control and reduction in incidence of distant metastases.

Bedford et al (2000), applied three-dimensional conformal radiotherapy techniques with chemotherapy to allow a better conformation of the treated volume. The potential benefit of conformal therapy is the possible dose escalation of the tumour site while avoiding adjacent radiosensitive structures, i.e. lung and spinal cord. Three different treatment plans were created and compared for a cohort of 10 patients. A two-phase treatment plan with conventional fields and blocks (designated by CV2), a two phase conformal plan (CF2) and a three-phase conformal plan (CF3), where the third phase was delivered to the GTV (gross tumour volume) only, were considered for each patient. It was observed that the CF2 technique reduced the volume of lung irradiated to 18 Gy from $19.7 \pm 11.8$ (1 SD) to $17.1 \pm 12.3 \%$, consequently permitting an increase in target dose to $59.1 \pm 3.2$ Gy without 
increasing the mean lung dose. It was observed that the technique CF3 facilitated a prescribed dose $60.7 \pm 4.3$ Gy to the target, for a spinal cord tolerance of $45 \mathrm{~Gy}$.

The patients with prostate cancer, considered within this study, have a high risk of pelvic nodal involvement or have radiological or pathological evidence of nodal metastases. IMRT has been shown to reduce normal tissue irradiation without sacrificing target coverage compared to conventional techniques (Nutting et al 2000 and Zelefsky et al 2000). Attempting to treat large volumes within the pelvis benefits from the sparing capabilities of IMRT (Nutting et al 2000). The small bowel is in close proximity to the pelvic nodes and the horse-shoe shape of the nodes, makes it a particularly difficult region to treat using conventional methods.

Intensity modulated radiation therapy (IMRT) may provide an improvement over conformal/conventional radiotherapy. IMRT treatment planning is usually performed independently of the delivery method, where the optimisation is performed based on a set of physical or/and biological constraints. When converting the plan, calculated by the planning system, into a deliverable sequence of leaf positions (MSF or DMLC) or compensator thicknesses, the dose delivered to the planning target volume and the organs at risk (OAR) may change depending on the leaf characteristics taken into account during the leaf sequencing. In the present study, a quantitative analysis of the impact of IMRT delivery effects for 3 or 5 fields, uniformly distributed around the patient, for multiple-static fields (MSF), dynamic MLC or compensator delivery techniques has been investigated. 
2. Method

\subsection{Overview of IMRT delivery techniques: MSF, DMLC or Compensator}

In converting the calculated fluence plan from the planning system into a deliverable sequence of leaf position (MSF or DMLC) or compensator thickness, different sequencing algorithms are used. In the case of the MSF technique, the Pinnacle planning system has a step-and-shoot K-means clustering algorithm (Hartigan 1975) that breaks the ideal fluence into smaller groups or clusters of equal value, in the case of the Elekta linac.

In the case of the compensator, the density of the compensation material, width, height, resolution for the compensator and depth of the plane for the compensator shape optimisation are defined. The energy fluence is then iteratively attenuated by the corresponding thickness of the modifier at each optimisation fluence point. A "granular compensator" was selected with spatial resolution of $0.05 \mathrm{~cm}$ and material density of $4.9 \mathrm{~g} / \mathrm{cm}^{3}$ (approximate density of standard compesator material). The source to compensator distance was $56.6 \mathrm{~cm}$ and the compensator was allowed a maximum thickness of $10 \mathrm{~cm}$.

In the case of the DMLC technique, the 'optimal' fluences are converted to the 'actual' fluences (Varian terms) using the leaf motion calculator (LMC) which designs the leaf motion patterns. The LMC takes into account the various MLC parameters such as maximum leaf span, leaf speed, transmission, rounded end effects and minimum leaf gaps. Since the $\mathrm{X}$ and $\mathrm{Y}$ jaws do not move during beam on, the maximum leaf span will determine how many carriage positions will be required to deliver the fluence for a given field width (X jaws). The field is split into multiple overlapping fields of the appropriate number of carriage or jaw positions. Although the leaf motions are not fully synchronised, the time of travel across the field is the same for all leaf pairs which helps to reduce the tongue-and groove effect (Essers et al 2001). 
2.2. Patient setup and treatment objectives

\subsubsection{Oesophagus Tumour Site and ADAC planning system}

A patient with oesophageal carcinoma was planned with 3 or 5 field IMRT plans (gantry angles: $0^{\circ}, 120^{\circ}, 240^{\circ}$ and $0^{\circ}, 72^{\circ}, 144^{\circ}, 216^{\circ}$ and $288^{\circ}$ ) using $\mathrm{P}^{3}$ IMRT (Pinnacle, ADAC version $6.0 \mathrm{~g}$ ). The clinical target volumes (CTV), spinal cord and lung parenchyma, were outlined on each image. The CTV region included both the oesophagus tumour and adjacent lymph nodes. The PTV region was generated by adding a three-dimensional margin of $15 \mathrm{~mm}$ to the CTV to account for movement and target definition uncertainties.

The goal of the plan was to deliver 55 Gy to the PTV, while maintaining the spinal dose less than or equal to $45 \mathrm{~Gy}$ and minimising the dose to the lungs. The spinal cord dose constraint of $45 \mathrm{~Gy}$ is conservative. Martel et al (1997) and Emami et al (1991) have shown that in head and neck cancers, the tolerance dose for the spinal cord is around $50 \mathrm{~Gy}$, with a 5\% chance of a complication occurring in 5 years. None of the patients treated by Martel et al (1997) developed radiation myelitis, with the spinal cord receiving doses up to $50 \mathrm{~Gy}$.

In addition to the spinal cord dose constraint, the volume of lung irradiated to $18 \mathrm{~Gy}$ has been used at the Royal Marsden NHS Trust. In the present study, no more than $20 \%$ of the lung could receive more than $18 \mathrm{~Gy}$. The prediction of lung complications at the treatment planning stage is not straightforward. There is no consensus on which dosimetric parameter should be used to reflect the clinical incidence of pneumonitis, however the volume of lung receiving $18 \mathrm{~Gy}$ was chosen since it is appropriately conservative (Martel et al 1994) to the present study. Cardiac radiation toxicity for

carcinoma of the oesophagus is not a major clinical concern because of the small number of long-term survivors. Therefore, the heart was not included as one of the OAR. 
2.2.2 Prostate and Pelvic Nodes Tumour Site and Helios/CadPlan planning system

Gantry angles of $180^{\circ}$ (posterior), $270^{\circ}$ (right lateral), $325^{\circ}$ (right anterior oblique), $35^{\circ}$ (left anterior oblique), $100^{\circ}$ (left posterior oblique) were chosen such that the 5 beams were spread out around the patient, provided good bowel sparing and were not opposing. The treatment was designed to deliver a dose of $70 \mathrm{~Gy}$ to the prostate and $50 \mathrm{~Gy}$ to the seminal vesicles and pelvic nodes. The prostate CTV was considered to be the entire visible prostate and was grown to a PTV with a $1 \mathrm{~cm}$ margin. However if the overlap between the PTV and rectum was large then the posterior margin was reduced to $8 \mathrm{~mm}$. The nodal CTV was expanded to a PTV with a uniform $5 \mathrm{~mm}$ margin. The goal dose constraints we have used for the prostate and pelvic node treatment are given in Clark et al (2002).

For prostate and pelvic node treatment with five gantry angles, typical beam lengths were 16$18 \mathrm{~cm}$ and beam widths were $10-18 \mathrm{~cm}$. Typical monitor units (MUs) were 95 (for a section of a split field) and 135 (for a maximum width single field). The prescribed dose was $2 \mathrm{~Gy}$ per fraction to the median of the prostate PTV (Clark et al 2002).

\subsection{Clinical impact of IMRT delivery effects}

An "optimum" IMRT plan was obtained with the planning system. The optimum fluence profiles were then leaf-sequenced in order to generate the leaf positions or compensator thicknesses, to allow the delivery of the planned profile. Each delivered IMRT plan would deliver a slightly different dose to the PTV, from that planned. The delivered IMRT fluence maps are then renormalised such as to attain the prescribed dose to the median of the principal PTV, i.e. 55 Gy for the oesophagus tumour and 70 Gy for the prostate tumour. In the case of the oesophagus tumour site, the impact of clustering the IMRT fluence profiles was studied, where each fluence map was divided into equal fluence levels, 
using a tolerance error method equivalent to that described in Bär et al (2001). The tolerance errors used for fluence clustering were $2 \%, 5 \%$ and $10 \%$.

In the case of the Helios/CadPlan planning system, several dose/volume values were compared before and after sequencing for the secondary PTV and critical organs (rectum, bladder, etc), for the case of 3 patients with prostate cancer and nodal involvement. These dose/volume values correspond to the dose delivered to $90 \%, 75 \%, 50 \%, 25 \%$ and $10 \%$ of the volume of the bladder, rectum and small bowel and 95\%, 75\%, 50\%, 25\% and 5\% of the volume of the PTV (prostate), right and left nodes. 
3. Results and discussion

3.1. Oesophagus tumour site and ADAC planning system

\subsubsection{Clinical impact of IMRT delivery effects}

An "optimum" IMRT plan was obtained with the ADAC planning system with a prescribed dose to the PTV region of $55 \mathrm{~Gy}$. The optimum profiles were then leaf-sequenced in order to generate the leaf positions or compensator thicknesses. These were then calculated to allow the delivery of the planned profile. Each delivered IMRT plan would deliver a different dose to the PTV, from that planned. These differences were in the order of $2-4 \%$ and were mainly due to head-scatter and transmission effects associated with the delivery technique, not accounted for during the planning stage. In order to compare the various delivery methods, the plans were all re-normalised such as to deliver a mean dose 55 Gy to the PTV.

The IMRT profiles were delivered using either the MSF or the compensator delivery techniques. In the case of the MSF technique, the fluence profiles were clustered into equal fluence levels, using a tolerance error method equivalent to that described in Bär et al (2001). The tolerance errors used were $2 \%, 5 \%$ and $10 \%$.

In figure 1, the DVH obtained for the delivered plans in case of 3 and 5 beams are compared to that planned for the PTV. In delivering the optimum fluence profile, the PTV dose has been "smeared" by both the MSF and the compensator techniques. In the case of the 3 beam delivery, the tolerance error of $2 \%$ has produced the dose distribution that better approximates the planned optimum (OPT_PTV). This is a consequence of the greater number of fluence levels allowed by the $2 \%$ than by the $5 \%$ and $10 \%$ cases.

In the case of the 5 beam plan, the delivered plans for the MSF for 5\% and $10 \%$ and the compensator are shown to "smear" the dose distribution planned for the PTV. The $2 \%$ is not shown due to hardware limitations not allowing the completion of the leaf-sequencing calculation. The compensator has produced the best delivered plan, of all the delivery methods represented for the 5 beam.

In figure 2, the dose distribution obtained on a CT slice of the patient for the 3 beam case, obtained for the (a) optimum plan, and MSF delivery with (a) 2\% and (c) 5\% tolerance error is shown. In figure $2 \mathrm{a}$, the $98 \%$ isodose curve conforms closely to the PTV region represented by the dark red circle are you going ot be able to publish in colour?. After delivery with the MSF technique, the high 
98\% isodose covers part of the adjacent lungs. In addition to this, the $80 \%$ isodose has significantly changed from the planned distribution (green curve in figure 2a) to the delivered (green curve in figure $2 \mathrm{~b} / \mathrm{c})$. Therefore, the lungs have received increased dose due to an increased head-scatter and transmission from the delivery technique. The spinal cord is also receiving an increased dose as may be observed comparing the 50\% isodose (blue)publishing colours ? curves in figure 2.

\subsubsection{Impact of IMRT delivery effects on organs at risk}

The DVH for the spinal cord is presented in figure 3 for both the 3 and 5 beam cases. In the case of the 3 beam, the spinal cord has received significantly more dose than initially planned, due to delivery effects. The increase in the maximum dose delivered to the spinal cord is of the order of 2-3 Gy, in addition to an overall increase of 5-10 Gy in the dose delivered to 50\% of the total volume of the spinal cord. In the case of the 5 beams, a similar increase in the spinal cord dose is observed, although, the DVH is different between the various delivery methods compared.

In figure 4 , the DVH obtained for the left and right lungs are presented. The maximum dose delivered to the lungs increases respectively by 1 and 3 Gy for the 3 and 5 applied beams. For the case of 5 applied beams, the dose delivered to $50 \%$ of the lung volume varies dramatic with the tolerance errors used to segment modulated beam profiles. There is no significant difference between the delivered dose to the lungs by the MSF technique for the $2 \%, 5 \%$ and $10 \%$ tolerance errors, in the case of the 3 beams. However, in the case of the 5 beams the delivered doses to the lungs by the MSF (5\% and $10 \%$ ) or the compensator are distinctly different. As shown in these results, the IMRT delivery techniques influence strongly the delivered dose distributions for the oesophagus tumour site, with the spinal cord and the lungs as organs at risk.

\subsection{Prostate and pelvic nodes and Helios planning system}

An "optimum" IMRT plan was obtained with the Helios planning system with a prescribed dose to the PTV region of 70 Gy to the primary prostate tumour and 48 Gy to the nodes. The optimum profiles were then leaf-sequenced in order to generate the leaf positions for the DMLC delivery. These were then calculated to allow the evaluation of the delivered profile and dose distribution. Each delivered IMRT plan would deliver a different dose distribution to the primary PTV, with subsequent increase in dose delivered to the secondary PTV (nodes) and organs at risk (bladder, small bowel and rectum). 
The mean dose (over the group of 3 prostate patients) delivered to the volumes of interest in "before" and "after" leaf-sequencing are presented in table 1, for the various dose/volume points studied. In the case of the organs at risk there is an increase of up to $3.3 \mathrm{~Gy}$ in the delivered dose to $50 \%$ of the total volume of the small bowel. While, the bladder and rectum are respectively subject to 2.15 Gy and 3.03 Gy more dose, delivered to $50 \%$ of their total volume. In the case of the small bowel and the rectum the increase in $3 \mathrm{~Gy}$ of delivered dose to $50 \%$ of the volume, may lead an increase in late radiation complication effects, rectal bleeding, etc. On the overall, the majority of the volumes of the organs at risk (bladder, small bowel and rectum) have received significantly more dose delivered to $90 \%$ of their volume. In the case of the rectum, this increase was the largest being of the order of 5 Gy to $90 \%$ of its total volume. In the case of the bladder and small bowel the increase was, respectively, 3.79 Gy and 3.14 Gy in the dose delivered to $90 \%$ of the total volume.

For the secondary PTV (right and left nodes), the leaf-sequencing of an IMRT "optimum" fluence profile (to be delivered by an MLC) leads to an average increase of, approximately, 2 Gy to $50 \%$ of the volume of the nodes, where the dose to the prostate tumour is not significantly altered. This increase in nodal dose may lead to late toxicity or complication effects. In addition to this, both nodes are also receiving more than 3 Gy dose delivered to the majority (90\%) of their volume. The increase is due mainly to head scatter and transmission radiation associated with the MLC delivery equipment and that is not accounted for by the Helios planning and optimisation planning system. This overall increase in the delivered dose is only observed for the volumes of interest: bladder, small bowel, rectum and nodes but not the primary PTV/prostate, to which everything is renormalised "after" leafsequencing. 


\section{Conclusions}

The impact of IMRT delivery effects on commercial treatment-planning systems such as the Pinnacle planning system (ADAC Laboratories Inc.) and CadPlan/Helios, (Varian Medical Systems) planning systems was evaluated. The study was performed for the oesophagus and prostate (with nodal involvement) tumours irradiated with multiple static fields, DMLC and the compensator delivery technique, after renormalising the delivered fluence such that all IMRT plans have the same mean PTV dose.

In the case of the oesophagus tumour site and using the Pinnacle planning system (ADAC), the IMRT delivery effects were shown to produce a smearing of the PTV (oesophagus) and an increase of 2-3 Gy in the dose delivered to the spinal cord. For the prostate (and pelvic nodes) tumour site and the CadPlan/Helios, (Varian Medical Systems) an increase of delivered dose of, approximately, 3 Gy was observed for $50 \%$ of the total volume of the organs at risk: bladder, rectum and small bowel. In addition to this, an increase of, approximately, 2 Gy was observed in the dose delivered the $50 \%$ of the total volume of the pelvic nodes, the secondary PTV region. The results obtained showed that if delivery effects are not accounted for at the planning/optimisation stage, then an increase in delivered dose to several of the volumes of interest may be expected, after the delivery of the IMRT profiles.

\section{Acknowledgements}

The work is supported by the Cancer Research Campaign [CRC] (under reference grant SP 2312|0201) and the Institute of Cancer Research. We are grateful to Dr Vivian Cosgrove and Dr Dave Convery for all the help associated with the experimental and software set-up, testing and the use of the interpreter, to Dr Alan Hounsell of Belvoir Hospital (Belfast) and Dr James Bedford for the helpful discussions and suggestions and to Dr Mark Phillips of the University of Washington (Seattle) for the data with the macro pencil beam model. 


\section{References}

Bär W, Alber M and Nusslin F 2001 A variable fluence step clustering and segmentation algorithm for step and shoot IMRT Phys. Med. Biol. 46 1997-2008

Bourland JD and Chaney EL 1992 A finite-size pencil beam model for photon dose calculations in three dimensions Med. Phys. 19, 1401-1412

Bortfeld TR, Burkelbach J, Boesecke and Schlegel W 1990 Methods of image reconstruction from projections applied to conformation radiotherapy Phys. Med. Biol. 35, 1423-1434

Bortfeld TR, Kahler D L, Waldron T J and Boyer A L 1994a X-ray field compensation with multileaf collimators Int. J. Rad. Oncol. Biol. Phys. 28 no.3 723-730

Bortfeld TR, Boyer AL, Schlegel W, Kahler DL and Waldron TJ 1994b Realisation and verification of three-dimensional conformal radiotherapy with modulated field Int. Jou. Rad. Oncol. Biol. Phys. 30, 899-908

Brahme A 1988 Optimization of stationary and moving beam radiation therapy techniques Radiother. Oncol. 12, 129-140

Carol MP 1992 An automatic 3D treatment planning and implementation system for optimised

conformal therapy by the NOMOS Corporation Proc. $34^{\text {th }}$ Ann. Meeting of the American Society for Therapeutic Radiology and Oncol. (San Diego 1992)

Carol MP 1994 Integrated 3D conformal multivane intensity-modulation delivery system for radiotherapy Proc. $11^{\text {th }}$ Int. Conf. on Computers in Radiotherapy (Manchester 1994) ed. AR Hounsell et al $172-173$

Cho PS and Marks II RJ 2000 Hardware-sensitive optimization for intensity modulated radiotherapy Phys. Med. Biol. 45, 429-440

Clark CH, Mubata CD, Meehan CA, Bidmead AM, Staffurth J, Humphreys ME, Dearnaley DP 2002 IMRT clinical implementation: Prostate and pelvic node irradiation using Helios and a 120-leaf MLC J. Appl. Clin. Med. Phys. Vol 3 (4) 273-284 2002 
Convery DJ and Webb S 1998 Generation of discrete beam-intensity modulation by dynamic multileaf collimation under minimum leaf separation constraints Phys. Med. Biol. 43, 2521-2538

Convery DJ, Cosgrove VP and Webb S 2000 Improving dosimetric accuracy of a dynamic MLC technique Proc. $13^{\text {th }}$ Int. Conf. On Computers in Radiotherapy (Heidelberg 2000) ed Schlegel W and Bortfeld TR 277-279

Essers M., de Langen M., Dirkx M. L. P. and Heijmen B. J. M. 2001. "Commissioning of a commercially available system for intensity-modulated radiotherapy dose delivery with dynamic multileaf collimation” Radiother Oncol. 60(2) p 215-224

Holmes T and Mackie TR 1994 A comparison of three inverse treatment planning algorithms Phys. Med. Biol. 39, 91-106

Holmes T W 2001 A method to incorporate leakage and head scatter corrections into a tomotherapy inverse treatment planning algorithm Phys. Med. Biol. 46, 11-27

Nutting C.M., Convery D.J., Cosgrove V.P., Rowbottom C., Padhani A.R. Webb S. and Dearnaley D.P. 2000. "Reduction of small and large bowel irradiation using an optimized intensity-modulated pelvic radiotherapy technique in patients with prostate cancer” Int J Radiat Oncol Biol Phys 48 (3) p $649-656$

Oldham M and Webb S 1995 The optimization and inherent limitations of 3D conformal radiotherapy treatment plans of the prostate Brit. Jour. Radiol. 68, 882-893

Seco J, Evans P and Webb S 2000 Modeling the effects of IMRT delivery: constraints and incorporation of beam smoothing into inverse planning Proc. $13^{\text {th }}$ Int. Conf. On Computers in Radiotherapy (Heidelberg 2000) ed Schlegel W and Bortfeld TR 542-544

Seco J, Evans P and Webb S 2001 Analysis of the effects of the delivery technique on an IMRT plan: comparison for multiple static field, dynamic and NOMOS MIMiC collimation PMB (in press)

Storchi P. R. M., van Battum L. J .and Woudstra E. 1999. "Calculation of a pencil beam kernel from measured photon beam data”. Phys Med Biol 44 (12) p 2917-28

Van Dalen S, Keijzer M, Huizenga H and Storchi P R M 2000 Optimization of multileaf collimator settings for radiotherapy treatment planning Phys. Med. Biol. 45, 3615-3625

Webb S 1989 Optimization of conformal radiotherapy dose distributions by simulated annealing Phys. Med. Biol. 34, 1349-1370 
Zelefsky M.J., Fuks Z., Happersett L., Lee H., Ling C.C., Burman C.M., Hunt M., Wolfe T., Venkatraman E.S., Jackson A., Skwarchuk M. and Leibel S.A. 2000. "Clinical experience with intensity modulated radiation therapy (IMRT) in prostate cancer" Radiother Oncol. 55 (3) p 241-249 


\section{$\underline{\text { Figure Captions }}$}

Figure 1. The DVH of the optimum (OPT_PTV) or delivered dose to the PTV by (a) 3 and (b) 5 IMRT plans. The delivery techniques represented are the (i) MSF with 2\% (red), 5\% (blue) and 10\% (green) tolerance error for the 3 beam and 5\% (blue) and 10\% (green) for the 5 beam and the (ii) compensator (orange) for the 5 beam case.

Figure 2. The oesophagus dose distribution obtained from the (a) planning system and delivered with MSF for (b) $2 \%$ and (c) 5\% tolerance error. The 98\%, 80\%, 50\% and 20\% iso-dose levels are respectively, red, green, blue and yellow.

Figure 3. The DVH obtained for the spinal cord in the case of the 3 and 5 beam plans. The delivery techniques represented are the (i) MSF with 2\% (red), 5\% (blue) and 10\% (green) tolerance error for the 3 beam and 5\% (blue) and 10\% (green) for the 5 beam and the (ii) compensator (orange) for the 5 beam case.

Figure 4. The DVH obtained for the left and right lungs in the case of the 3 and 5 beams IMRT plan. 
Table 1:

\begin{tabular}{|c|c|c|c|c|c|c|}
\hline & & & $\begin{array}{r}\text { MEA } \\
\text { FORE” LE }\end{array}$ & $\begin{array}{l}\text { SSE } \\
\text { EQUENCIN }\end{array}$ & & \\
\hline - & & DOS & ELIVEREI & VOLUMES O & EREST & \\
\hline VOL & BLADDER & BOWEL & RECTUM & $\begin{array}{c}\text { PTV } \\
\text { PROSTATE }\end{array}$ & $\begin{array}{l}\text { RIGHT } \\
\text { NODE }\end{array}$ & $\begin{array}{l}\text { LEFT } \\
\text { NODE }\end{array}$ \\
\hline $5 \%$ & & & & 71.00 & 53.33 & 65.33 \\
\hline $10 \%$ & 69.73 & 41.17 & 68.00 & & & \\
\hline $25 \%$ & 62.37 & 31.50 & 51.00 & & 50.17 & 50.50 \\
\hline $50 \%$ & 48.83 & 24.17 & 40.83 & 70.00 & 48.30 & 48.30 \\
\hline $75 \%$ & 39.70 & 13.67 & 36.10 & & 46.43 & 46.50 \\
\hline $90 \%$ & 32.07 & 2.67 & 31.17 & & & \\
\hline $95 \%$ & & & & 67.33 & 43.67 & 43.83 \\
\hline
\end{tabular}

\begin{tabular}{|c|c|c|c|c|c|c|}
\hline & & & $\begin{array}{r}\text { MEA } \\
\text { TER" LEA }\end{array}$ & $\begin{array}{l}\text { SEE } \\
\text { QUENCING }\end{array}$ & & \\
\hline . & & DOS & ELIVEREI & VOLUMES O & EREST & \\
\hline VOL & BLADDER & BOWEL & RECTUM & $\begin{array}{c}\text { PTV } \\
\text { PROSTATE }\end{array}$ & $\begin{array}{l}\text { RIGHT } \\
\text { NODE }\end{array}$ & $\begin{array}{l}\text { LEFT } \\
\text { NODE }\end{array}$ \\
\hline $5 \%$ & & & & 72.28 & 53.62 & 63.14 \\
\hline $10 \%$ & 68.24 & 41.80 & 66.16 & & & \\
\hline $25 \%$ & 61.85 & 33.74 & 52.46 & & 51.62 & 51.60 \\
\hline $50 \%$ & 50.98 & 27.49 & 43.86 & 69.77 & 50.34 & 49.98 \\
\hline $75 \%$ & 42.59 & 15.24 & 39.34 & & 48.90 & 48.56 \\
\hline $90 \%$ & 35.85 & 5.73 & 36.23 & & & \\
\hline $95 \%$ & & & & 66.36 & 46.76 & 46.48 \\
\hline
\end{tabular}


Figure 1:
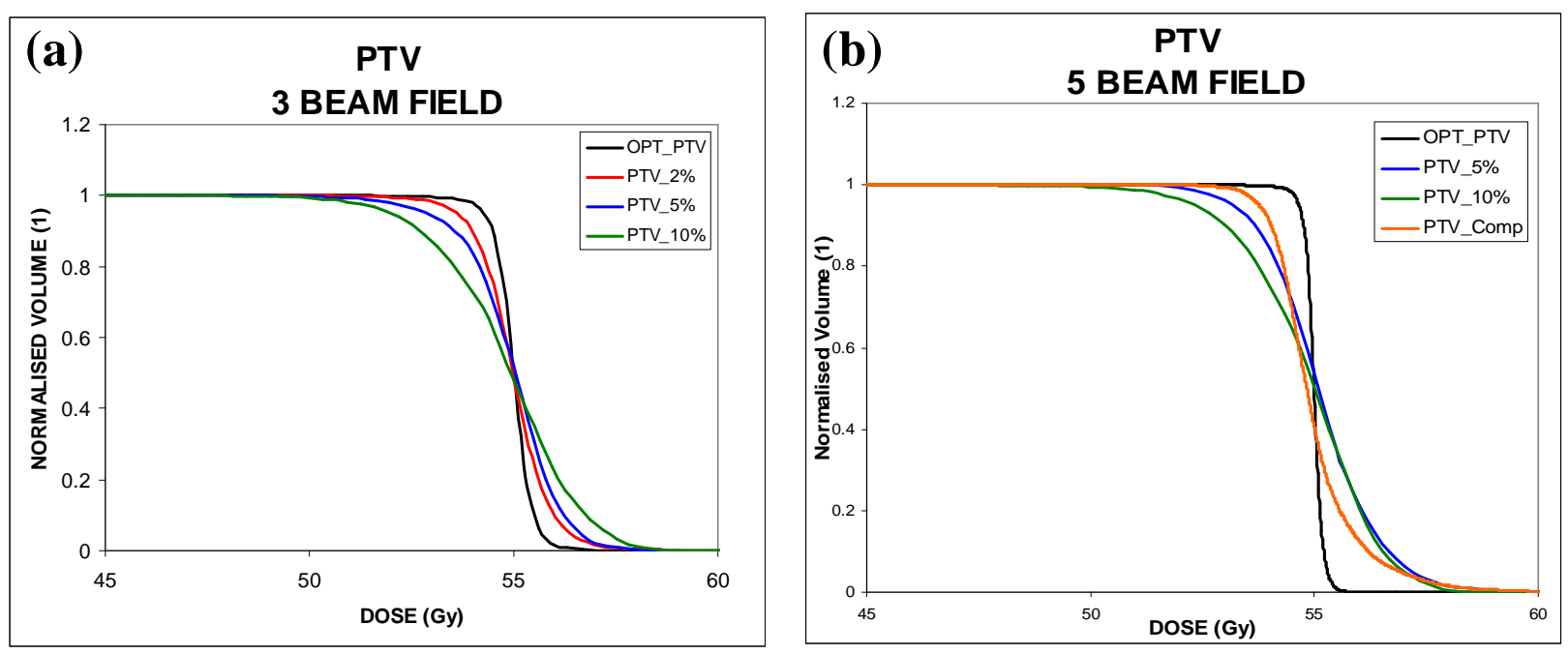
Figure 2

(a)

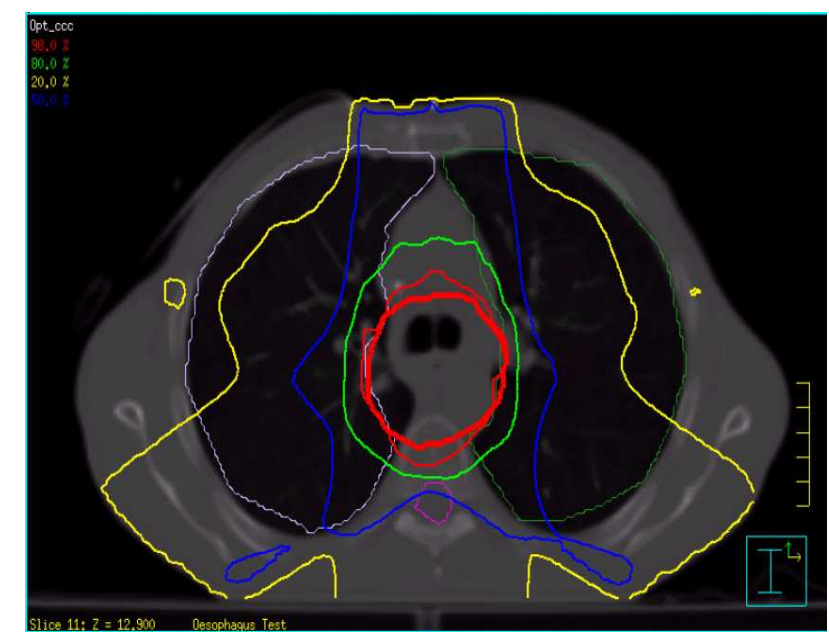

(b)

(c)

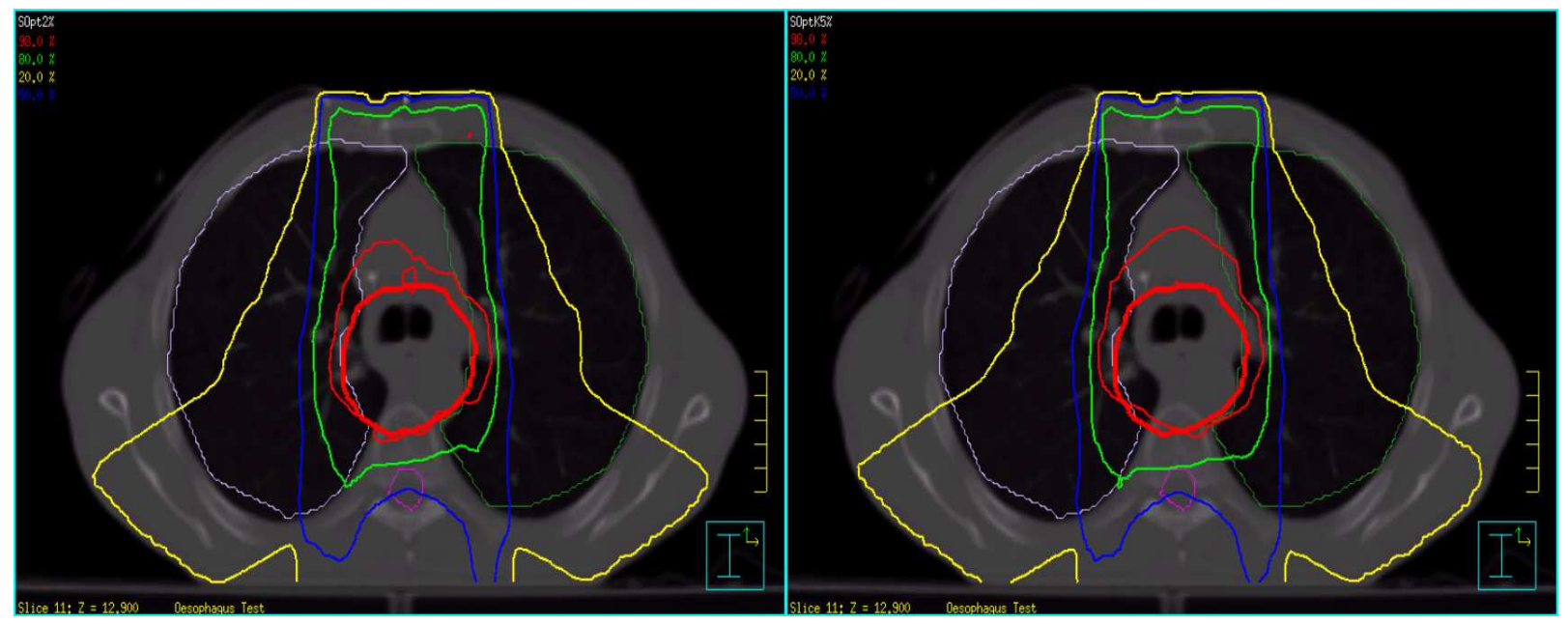


Figure 3
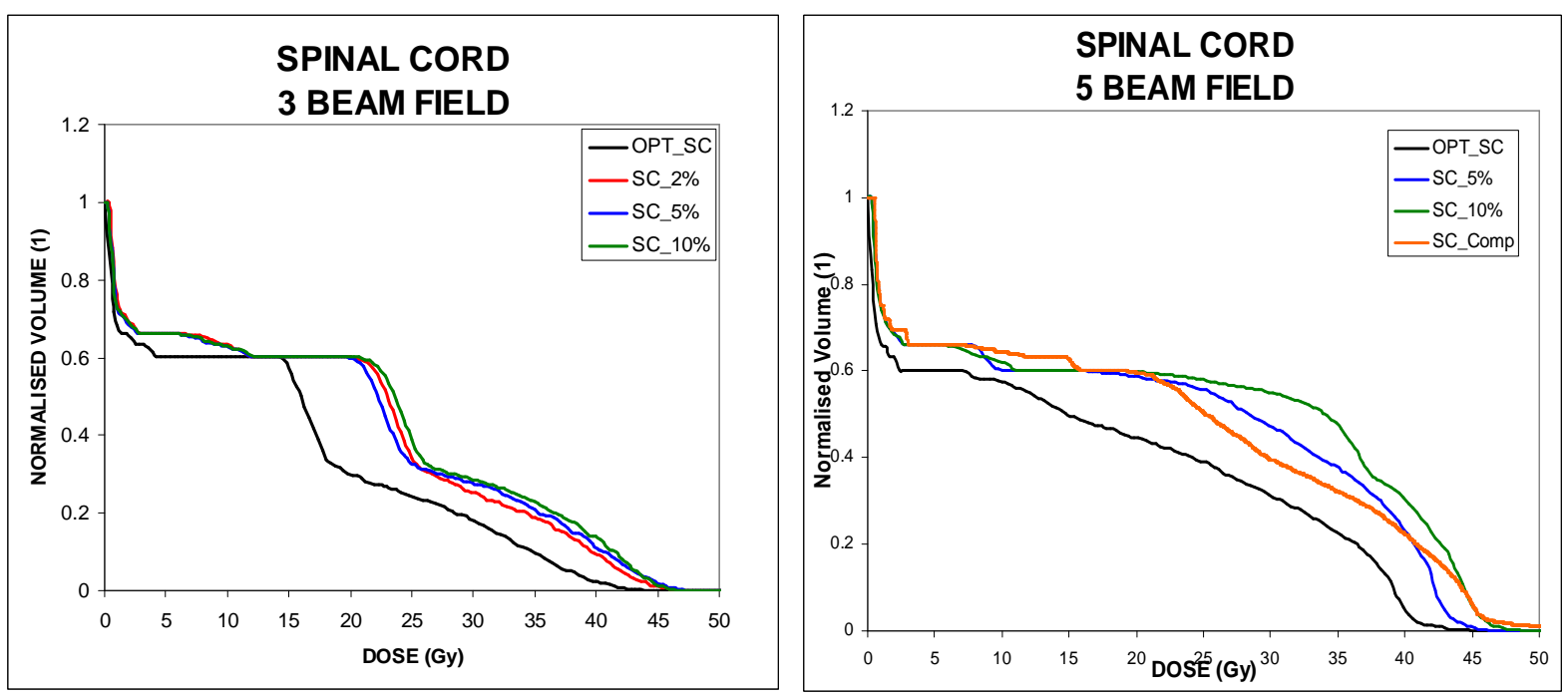
Figure 4
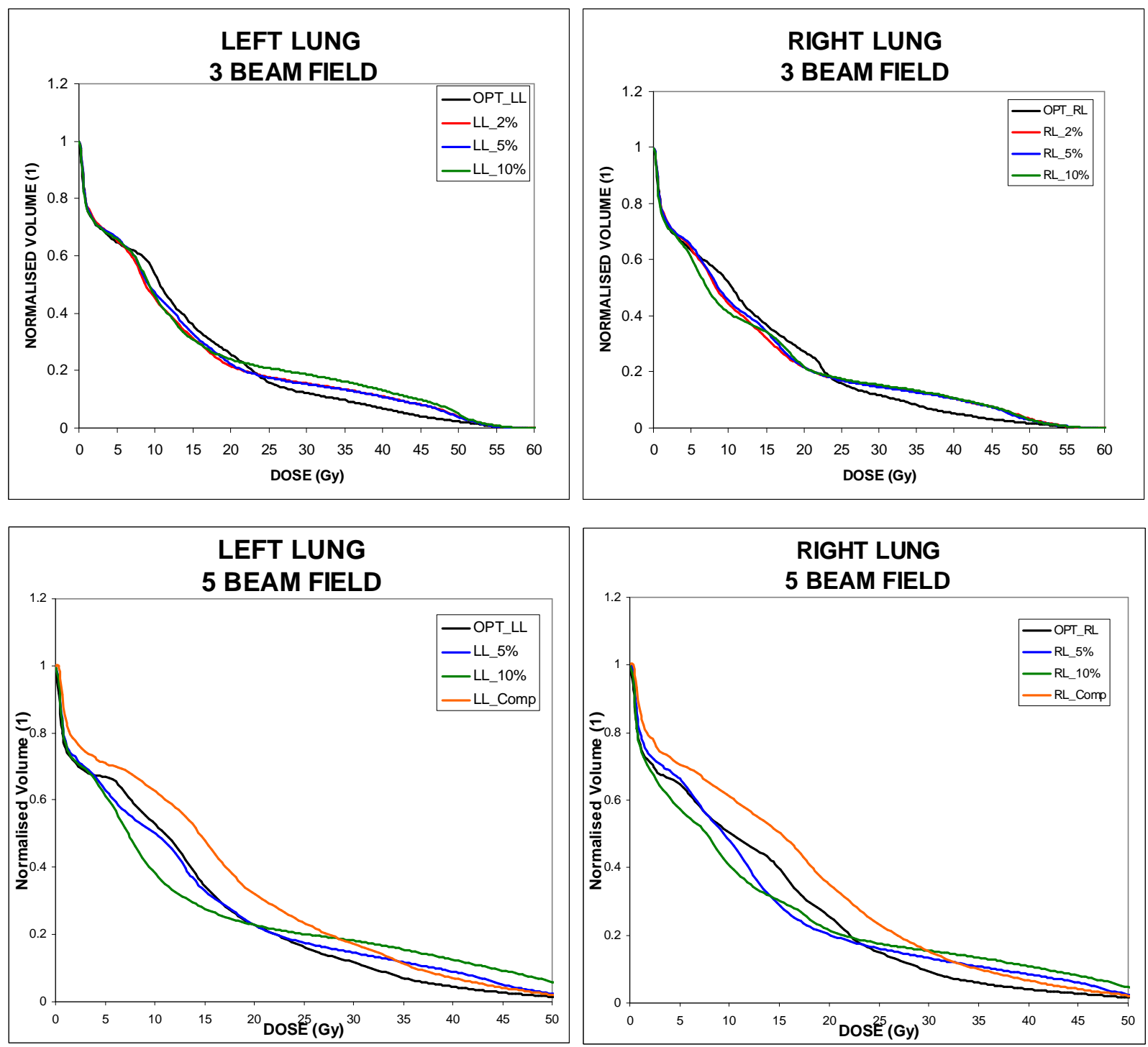